\title{
Bespoke 3D-Printed Polydrug Implants Created via Microstructural Control of Oligomers
}

\author{
Laura Ruiz-Cantu, Gustavo F Trindade, Vincenzo Taresco, Zuoxin Zhou, Yinfeng He, \\ Laurence Burroughs, Elizabeth A. Clark, Felicity R.A.J. Rose, Christopher Tuck, Richard Hague, \\ Clive J. Roberts, Morgan Alexander, Derek J. Irvine,* and Ricky D. Wildman*
}

Cite This: https://doi.org/10.1021/acsami.1c07850

Read Online

ACCESS | Lلlll Metrics \& More | 回 Article Recommendations | S1 Supporting Information

ABSTRACT: Controlling the microstructure of materials by means of phase separation is a versatile tool for optimizing material properties. Phase separation has been exploited to fabricate intricate microstructures in many fields including cell biology, tissue engineering, optics, and electronics. The aim of this study was to use phase separation to tailor the spatial location of drugs and thereby generate release profiles of drug payload over periods ranging from 1 week to months by exploiting different mechanisms: polymer degradation, polymer diluent dissolution, and control of microstructure. To achieve this, we used drop-on-demand inkjet three-dimensional (3D) printing. We predicted the microstructure resulting

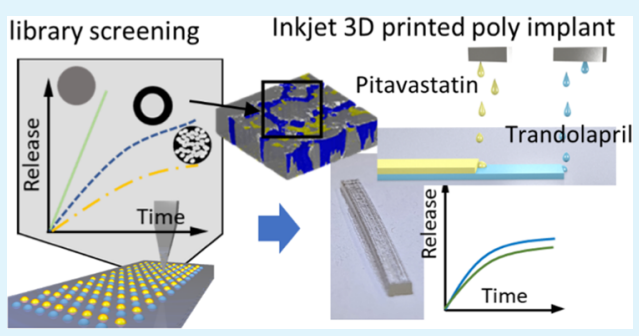
from phase separation using high-throughput screening combined with a model based on the Flory-Huggins interaction parameter and were able to show that drug release from 3D-printed objects can be predicted from observations based on single drops of mixtures. We demonstrated for the first time that inkjet 3D printing yields controllable phase separation using picoliter droplets of blended photoreactive oligomers/monomers. This new understanding gives us hierarchical compositional control, from droplet to device, allowing release to be "dialled up" without manipulation of device geometry. We exemplify this approach by fabricating a biodegradable, long-term, multiactive drug delivery subdermal implant ("polyimplant") for combination therapy and personalized treatment of coronary heart disease. This is an important advance for implants that need to be delivered by cannula, where the shape is highly constrained and thus the usual geometrical freedoms associated with $3 \mathrm{D}$ printing cannot be easily exploited, which brings a hitherto unseen level of understanding to emergent material properties of 3D printing.

KEYWORDS: 3D printing, drug release, implants, phase separation, inks

\section{INTRODUCTION}

Personalized medicine is often described as providing the right patients, with the right drug, at the right dose, at the right time. ${ }^{1}$ Over recent years, we have seen an evolution toward personalized medicine with impressive improvements in treatment outcomes that have been driven by the scientific and technological advancements in genomic sequencing and bioinformatics. These advances have shown that people respond differently to treatments due to the intricacies of inter- and intraindividual variabilities. ${ }^{2}$ Delivering personalized medicine requires technological innovations in drug delivery systems that meet the demand for customizable therapy to achieve maximal treatment efficacy with minimal side effects. ${ }^{3}$ To achieve the desired personalization, formulations and fabrication production processes need to have a range of flexibility to be tuned on demand to meet the patients' needs and three-dimensional (3D) printing offers a route to this personalization, through its design freedoms and flexible multimaterial manufacturing approach that can be tuned on demand. ${ }^{4}$

$3 \mathrm{D}$ printing affords reproducible creation of personalized pharmaceutical dosage forms by utilizing precise control of factors such as droplet size ${ }^{5}$ or filament size to control the dose $^{6}$ and compartmentalization of multiple APIs, which allows the creation of complex drug release profiles. ${ }^{4,7}$ Several multimaterial 3D-printing methods have been used to demonstrate drug delivery, including fused deposition modeling, ${ }^{8}$ binder jetting, ${ }^{9}$ selective laser sintering, ${ }^{10}$ and ink jet printing. ${ }^{11}$ However, 3D printing faces challenges. There are not always the necessary materials available, and even if ondemand production is possible, it is difficult for an end user to know exactly which material or material combination will result in the desired outcome. Further, sometimes is not possible to easily exploit design freedoms due to limitations in the implementation of a device. A pertinent example is the long-term subdermal implants delivered by cannula, which

Received: April 28, 2021

Accepted: July 9, 2021 
Scheme 1. Schematic Diagram of the Design System ${ }^{a}$

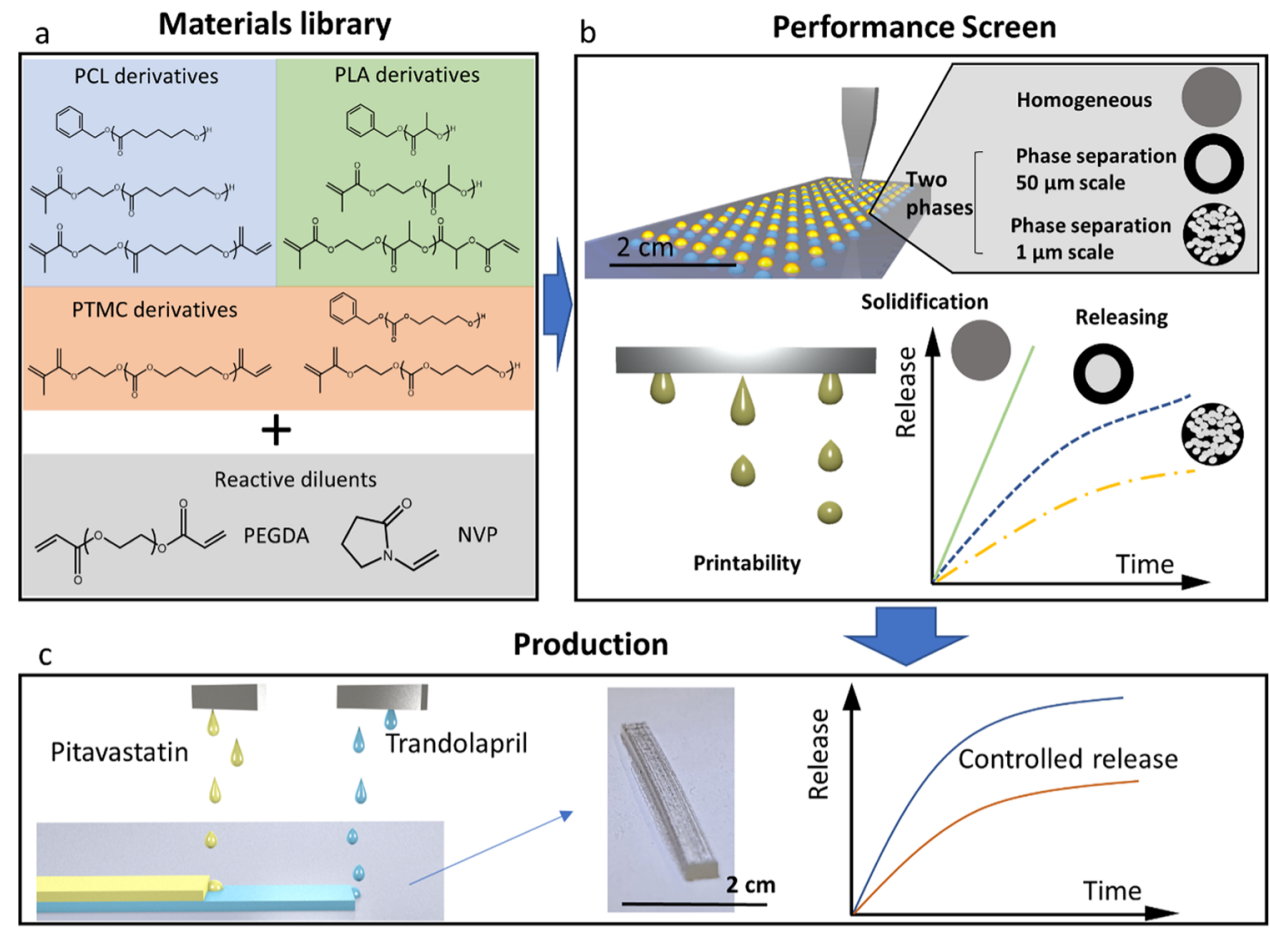

a (a) Material library formed by the biodegradable oligomers PCL, PLA, and PTMC derivatives with - OH, MA, and MA-A end functionalities) and reactive solvents (PEGDA and NVP). Abbreviations: poly(caprolactone) (PCL), poly(D,L-lactic acid) (PLA), poly(trimethylene carbonate) (PTMC), poly(ethylene glycol diacrylate) (PEGDA), $N$-vinylpyrrolidone (NVP), hydroxyl end terminated (-OH), mono methacrylate (MA) functionalized, and dual acrylate-methacrylate (MA-A) functionalized. (b) Performance screen. (c) Fabrication of the tuneable drug subdermal drug delivery implants containing multiple actives for the treatment of coronary heart disease.

have restrictions on shape and size. To overcome these challenges, we have turned to inkjet-based 3D printing, seeking to develop drug delivery depots that can be "dialled up" to achieve specified release profiles. We posit that it is possible to go from material generation, selection, characterization and manufacture in a rational sequence, with the drug delivery outcomes predictable from material understanding and highthroughput screening.

Drug depots, e.g., the hormonal contraceptive implant and others $^{12-15}$ must meet clinical demands/benefits associated with their use, such as temporal control of the payload release for hours, days, and months, minimization of frequent repeat dosing, ${ }^{16}$ and capable of being delivered subcutaneously via a cannula-the implants must fit a design envelope with a maximum diameter of $2 \mathrm{~mm}$ and a length between 1 and $4 \mathrm{~cm}$. In addition, we intend for an implant that will be active for greater than 6 months, degradable, and customizable for variation in patient weight, sex, age, genetic profile, and length of treatment. ${ }^{17,18}$ These challenges require the development of materials and an understanding of the behavior within the manufacturing process. Consequently, in this work, we develop a library of $3 \mathrm{D}$ printable, degradable, and potentially drug release materials, from which combinations of materials can be chosen to select desired drug release profiles (Scheme 1). Since inkjet printing is a drop-by-drop deposition method, we developed a screening method based on observable phaseseparation behavior in a single drop and extended that to macroscopic structures via the Flory-Huggins interaction parameter. From this, we then set out to exploit various mechanisms available for affecting drug release: polymer degradation rate, diluent dissolution rate, and phase separation via the development of a library of formulations/inks that can be selected to enable the "dial up" of release from a multiple drugs' loaded 3D-printed implant. Finally, we exemplify our approach by fabricating a multidrug implant with release profiles that are tuneable through the choice of material combinations.

\section{RESULTS AND DISCUSSION}

2.1. Material Synthesis and Material Library. The control of drug release from a 3D-printed implant can be exerted via control over its composition and microstructure. ${ }^{19}$ To control the composition, first we chose three core materials, poly(caprolactone) (PCL), poly(lactic acid) (PLA), and poly(trimethylene carbonate) (PTMC), which offer a range of degradation behaviors. Second, we combined our materials with diluents, $n$-vinylpyrrolidone (NVP) and poly (ethylene glycol diacrylate) $250 \mathrm{M}_{\mathrm{w}}$ (PEGDA), that offer differences in drug affinity and dissolution behavior. Finally, we synthesized core materials into low-molecular-weight $(5 \mathrm{kDa})$ biodegradable oligomers with the following head-terminal group combinations, benzyl-hydroxyl (Bz-OH), methacrylatehydroxyl (MA-OH), and methacrylate-acrylate (MA-A) (Scheme 1 and Figure S1), allowing us to influence phase separation when combined with diluents. Our choice of core materials was informed by their existing approvals and use in the biomedical industry and selected for reduced legislative barriers to adoption compared to completely new materials. ${ }^{20}$ Furthermore, oligomers were synthesized by ring-opening polymerization using metal-free organocatalysis, ${ }^{21}$ chosen to minimize the toxicological impact of any subsequent medical devices. ${ }^{22}$ 
a $P C L \bigcirc P L A \bigcirc P T M C \bigcirc P E G D A \varnothing P V P Q$
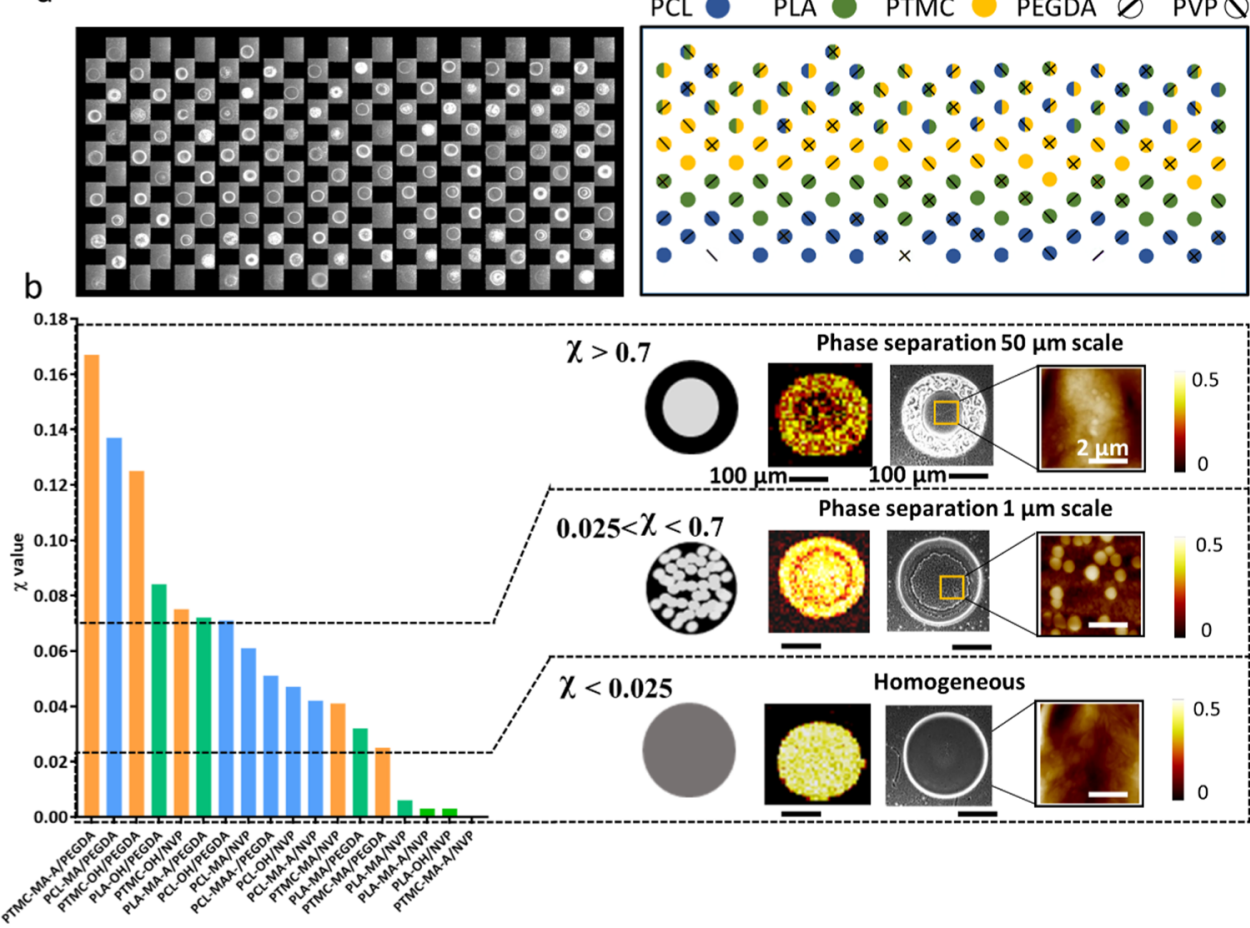

Figure 1. Phase-separation screening on single droplets of materials. (a) Optical microscopy images of droplets on a microarray format and schematic representation of the microarray (b) ToF SIMS images, microscopy images, and topographical AFM images representing different types of phase separation observed on the materials. PCL-MA/PVP represents phase separation with $50 \mu \mathrm{m}$ scale features, PTMC-MA/PVP represents phase separation with $1 \mu \mathrm{m}$ scale features, and PLA-MA/PVP represents homogenous samples. ToF SIMS images represent negative ions PCL-MA $\left(\mathrm{C}_{4} \mathrm{H}_{5} \mathrm{O}_{3}^{-}\right)$, PTMC-MA $\left(\mathrm{C}_{6} \mathrm{H}_{9} \mathrm{O}_{2}^{-}\right)$, and PLA-MA $\left(\mathrm{C}_{3} \mathrm{H}_{3} \mathrm{O}_{2}^{-}\right)$, respectively. The bright band around the rim of the spots on the microscopy images, second and third from top to bottom, are optical artifacts. The phase-separation taxonomy graph is based on $\chi$ values and different phaseseparation morphologies from the spots on the microarray.

The library was created by combining nine hydrophobic oligomers in a 1:1 ratio with two relatively hydrophilic reactive solvents/diluents, giving a total of 18 inks (Scheme 1). Poly(ethylene glycol) (PEG)-based materials are some of the most efficient plasticizers used for polymers ${ }^{23-25}$ due to advantages such as a broad range of molecular weights, nontoxicity, and good miscibility. The plasticization of PEG can effectively increase the chain mobility of some polymers and improve their ductility and drawability, thus broadening the range of printability in our application. We selected the diacrylate version of PEG so it could act as a plasticizer and reactive solvent at the same time. It is also nondegradable, so it will not be depleted from the structure. Meanwhile, NVP was selected because once polymerized into poly ( $N$-vinylpyrrolidone) (PVP), it has the ability to form a water-soluble composite structure with insoluble active substances and improve the release and solubility of drugs. ${ }^{18,26}$ After degradation studies, we identified that the structures formed with just NVP had a weak structural integrity and thus, from then on, combined NVP with PEGDA to improve the mechanical performance of our printed formulations.

2.2. Screening. 2.2.1. Predicting Phase Separation. Having established a library of materials with the potential to deliver a range of differing microstructures from our formulation combinations, we characterized the emergent behavior via a single-drop screening (SDS) method. This method was based on microarrays of single drops (10 pL) rapidly deposited and cured on a glass slide using an automated microarray printer. ${ }^{27}$ Phase-contrast optical images were taken of each $200 \mu \mathrm{m}$ spot (Figure 1a), and the surface chemistry and microstructure/phase-separation properties were further evaluated using optical microscopy, time-of-flight secondary ion mass spectrometry (ToF SIMS), automated peak force quantitative nanomechanics (QNM), and atomic force microscopy (AFM). This analytical combination provided information on the phase separation and oligomer distribution data (Figures $1 \mathrm{~b}$ and $\mathrm{S} 2$ ) required to create a phase-separation "taxonomy." This data was then used to inform a predictive model based on the Flory-Huggins interaction parameter $(\chi)$, which we proposed to use to determine the likelihood of phase separation (see the Supporting Information, Tables S1 and S2) (Figure 1b).

The Flory-Huggins parameter describes the excess free energy of mixing and governs phase behavior for polymer blends and block copolymers. ${ }^{27}$ For chemically dissimilar polymers, it can be assumed that the enthalpy is the dominant contribution to the Gibbs free energy, and thus $\chi$ (which can take negative or positive values) determines the mixing state. For nonpolar polymers, this term is taken to describe the mismatch in cohesive energy density between different monomer units. A large mismatch in cohesive energy density results in a larger magnitude in $\chi$ and, hence, a greater tendency for demixing. ${ }^{28}$ To calculate the $\chi$ parameter of different combinations of oligomers, we used the Hansen solubility parameter (HSP) of each individual component, which is drawn from their dispersion $(\delta D)$, polarity $(\delta P)$, and hydrogen bonding $(\delta H)$ values. The closer the HSP value of the molecules are to each other, the more alike they are and the more likely they are to be miscible. ${ }^{29,30}$ The correlation between the $\chi$, HSP, and the oligomers/solvents can be 
a

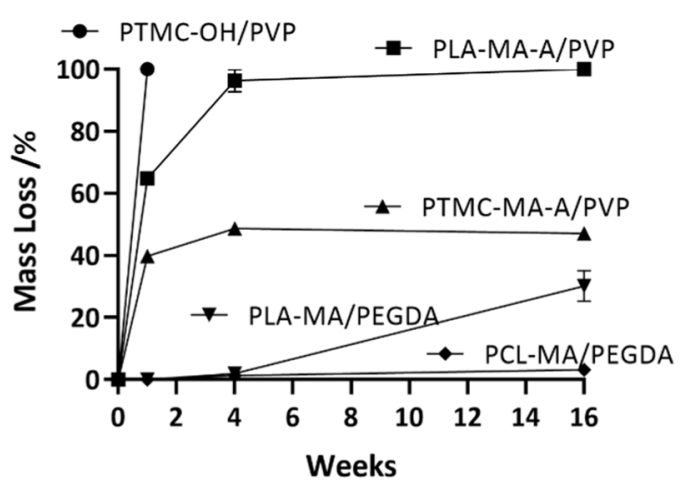

b
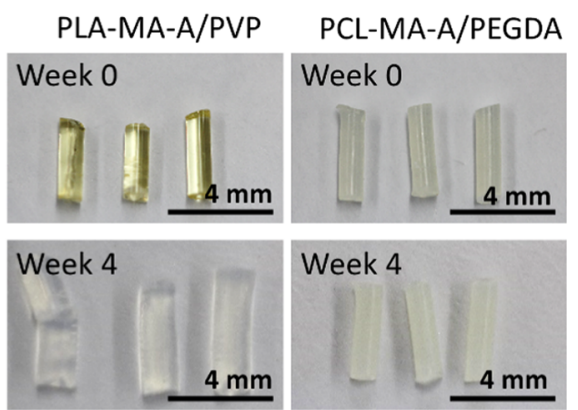

C
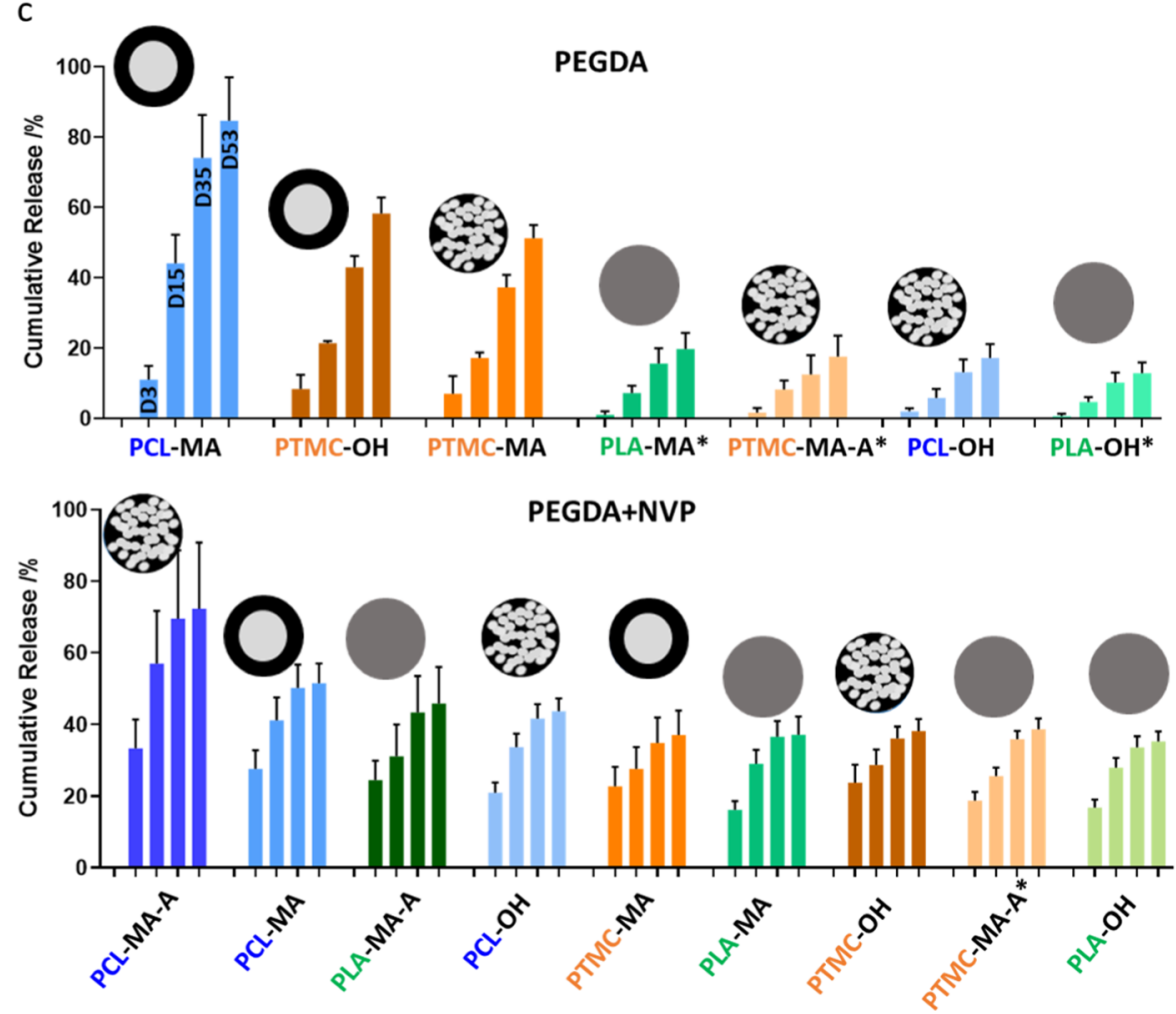

Figure 2. Materials screening on cast samples. (a) Representative results of different degradation profiles observed on the 18 formulations $(n=3$, $\pm S D$ ). (b) Representative images of the macroscopic changes observed after 4 weeks of degradation. (c) Cumulative release of trandolapril from oligomer: PEGDA/PVP samples after $3,15,35$, and 53 days $(n=3, \pm \mathrm{SD})$. The schematics in the graphs represent the phase separation on the materials observed on the droplet screening. The oligomers (PTMC-MA-A and PLA-OH) marked with an asterisk are those that the $\chi$ values showed that the drug has more affinity for the oligomer rather than the diluent, therefore will stay with the oliger when phase separation ocurrs. Data are presented as mean values $\pm \mathrm{SD}$.

exemplified with the material combinations PTMC-MA-A/ PEGDA and PTMC-MA-A/NVP, which have the highest and the lowest $\chi$ values, respectively. The calculated HSP of individual components were PTMC-MA-A 20.8, PEGDA 18.5, and NVP 20.8 (Table S1), showing that PTMC-MA-A and NVP are more alike, and therefore, no phase separation is favored.

Our observations indicated that the polymer spots exhibit three different types of microstructures: (a) two phases with domains of $50 \mu \mathrm{m}$ size, (b) two phases with domains of $1 \mu \mathrm{m}$ size, and (c) homogeneous spots (Figures $1 \mathrm{~b}$ and S2). ${ }^{31}$ Consequently, materials were ordered according to their $\chi$ values and compared to the observed microstructure taxonomy (Figure 1b). Broadly, the material microstructure conformed to classification by $\chi$ and approximate $\chi$ values demarking boundaries between microstructure types were identified: (a) below 0.025 presented homogenous distribution, (b) with values between 0.025 and 0.07 presented phase separation with $1 \mu \mathrm{m}$ features, and (c) above 0.07 exhibited phase separation with $50 \mu \mathrm{m}$ features. Some of them (PTMC/PVP, PTMCMA/PVP, and PTMC-MA/PVP/PEGDA) presented both phase separation at 1 and $50 \mu \mathrm{m}$ scales, which reflected the fact that the boundaries were not distinct and could be influenced by other physical properties, such as viscosity and curing rate. The boundaries were chosen such that we included all of the samples that exhibited $1 \mu \mathrm{m}$ features in one classification. Consequently, we estimated the likely error in the boundary by calculating the average difference between $\chi$ at the boundary and at the exceptions, resulting in an approximate error of \pm 0.01 . 
a
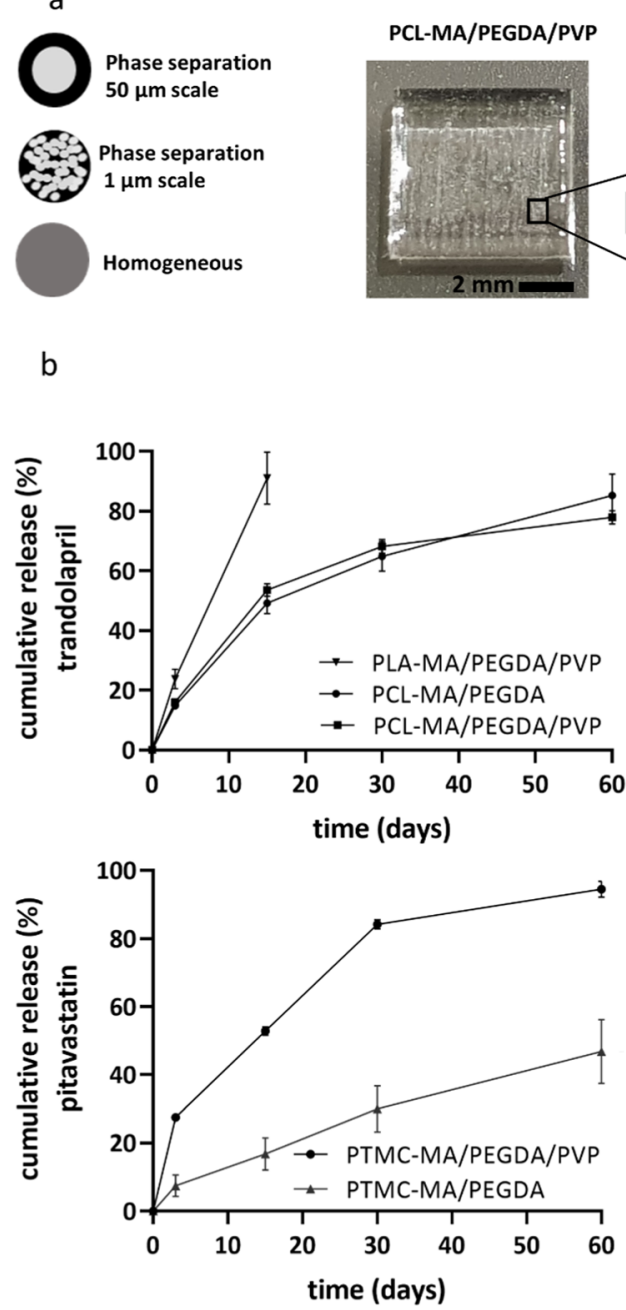

c

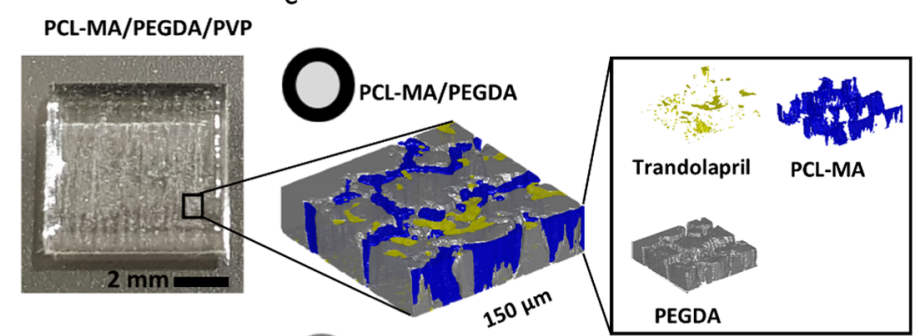

PLA-MA/PEGDA/PVP
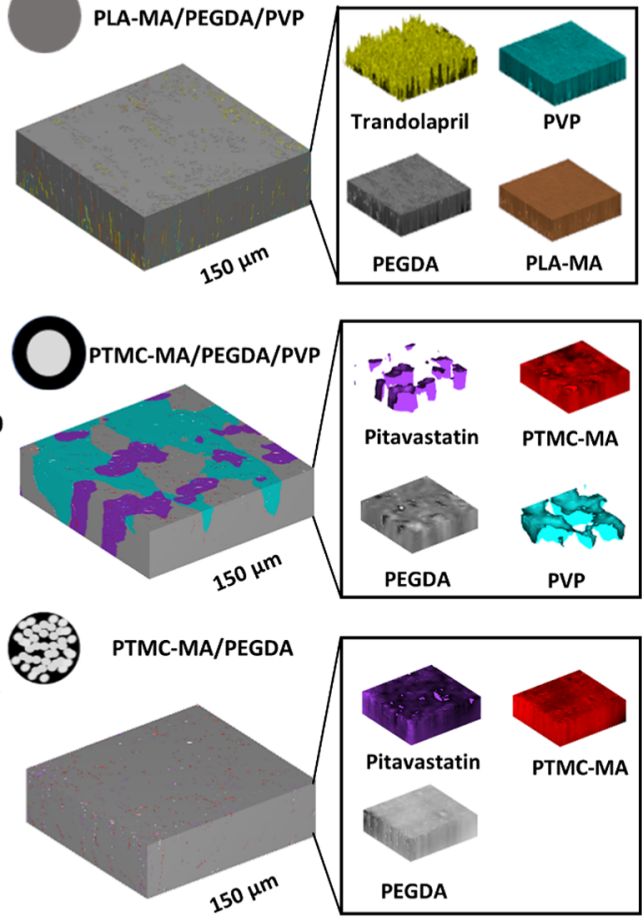

Figure 3. 3D-printed formulations containing the API trandolapril and pitavastatin with tuned release profiles. (a) Schematic of different phaseseparation morphologies and representative image of the 3D-printed PCL-MA/PEGDA/PVP formulation. (b) Release profiles of different 3Dprinted formulations containing trandolapril and pitavastatin for a period of 60 days $(n=3, \pm \mathrm{SD})$. The asterisk represents the component of the ink that trandolapril or pitavastatin has more affinity with according to the $\chi$ values. (c) $3 \mathrm{D}$ reconstruction of ToF SIMS depth profiles, showing that the phase separation represents the negative ions PCL-MA $\left(\mathrm{C}_{4} \mathrm{H}_{5} \mathrm{O}_{3}^{-}\right)$, PLA-MA $\left(\mathrm{C}_{3} \mathrm{H}_{3} \mathrm{O}_{2}^{-}\right)$, PTMC-MA $\left(\mathrm{C}_{6} \mathrm{H}_{9} \mathrm{O}_{2}^{-}\right)$, PEGDA $\left(\mathrm{C}_{2} \mathrm{H}_{3} \mathrm{O}^{-}\right)$, PVP $\left(\mathrm{C}_{4} \mathrm{H}_{6} \mathrm{NO}^{-}\right)$, trandolapril $\left(\mathrm{C}_{24} \mathrm{H}_{33} \mathrm{~N}_{2} \mathrm{O}_{5}^{-}\right)$, and pitavastatin $\left(\mathrm{C}_{20} \mathrm{H}_{15} \mathrm{FN}^{-}\right)$. Data are presented as mean values $\pm \mathrm{SD}$.

2.2.2. Degradation Rate. To determine the influence of microstructure on the cured ink's macro-material/degradation, cast cylinders (length $4 \mathrm{~mm}$, radius $0.5 \mathrm{~mm}$ ) of the 18 inks were prepared and subjected to degradation studies. The duration of degradation was 16 weeks, and the mass loss rates are shown in Figures 2 and S3. As most of the structures printed with NVP did not maintain their integrity following curing, we replaced the NVP only formulations with PEGDA/ NVP solvent combinations. These were used in the biocompatibility (Figure S5) and drug release studies to enable faster degradation than PEGDA alone while maintaining some structural integrity.

2.2.3. Drug Release. To gauge the drug releasing propensity of our ink, an angiotensin-converting enzyme (ACE) inhibitor, trandolapril, was used as a model drug in the drug release screening from candidate formulations. For this study, we used the same sample shape as for the degradation study. The drug release profile was screened for a period of 8 weeks on 16 of the formulations, with PCLMAA/PEGDA and PLAMAA/ PEGDA being eliminated as they were not within the printability range (Figure S4 and Tables S4 and S5). With
PEGDA alone, Figure 2c, we observed that the fastest release profiles were those from PCL-MA/PEGDA and PTMC-OH/ PEGDA, each of which presented a phase separation with 50 $\mu \mathrm{m}$ feature microstructure in the SDS. PTMC-MA/PEGDA exhibited the next fastest release and presented a phase separation with a microstructure containing $1 \mu \mathrm{m}$ features. In each of these cases, $\chi$ analysis predicted that trandolapril had more affinity for and would migrate toward the PEGDA (Table S3). In contrast, those materials that had a significantly slower release had a propensity for homogeneous microstructure and, additionally, a greater drug affinity for oligomers over PEGDA. This suggests that the phase separation leads to a structure whereupon the drug has a relatively easy path to escape from the implant, but that when no phase separation occurs, the mixture is a relatively hydrophobic material with a low permeability and exhibits slow drug release. As we will see when dissecting 3D-printed structures, when phase separation occurs, there results a network, and it is this structure that affords the drug a faster release from the implant.

Using NVP to form PEGDA/PVP-based mixtures (Figure 2c) leads to very similar dissolution rates across all of the 


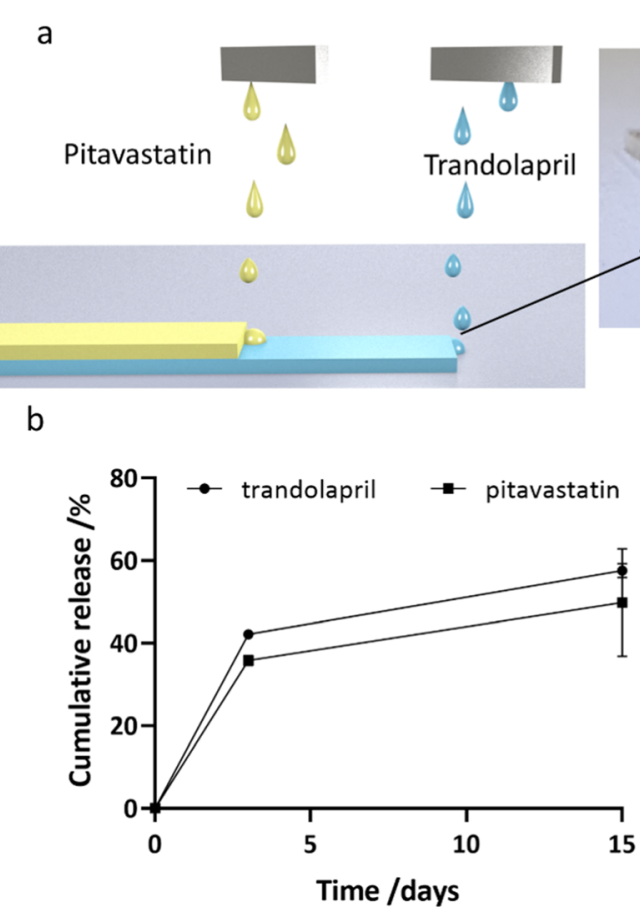

C

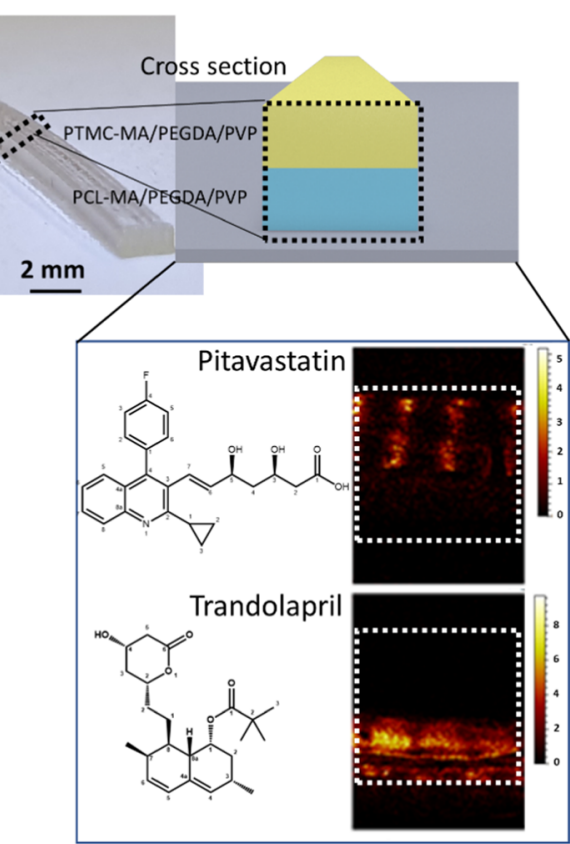

Figure 4. 3D-printed multidrug implant exemplar for the co-delivery of the drugs pitavastatin (top) and trandolapril (bottom). (a) Schematic representation of the implant showing the relative positions of drug-loaded portions and image of the 3D-printed implant showing its appearance. (b) Cumulative drug release of both drugs over 5 and 15 days $(n=3, \pm \mathrm{SD})$. (c) ToF SIMS images on the implant cross section representing the negative ions of pitavastatin $\left(\mathrm{C}_{20} \mathrm{H}_{15} \mathrm{FN}^{-}\right)$on the top image and trandolapril $\left(\mathrm{C}_{24} \mathrm{H}_{33} \mathrm{~N}_{2} \mathrm{O}_{5}{ }^{-}\right)$on the bottom image. The images show the vertical drug's distribution through the implant. Data are presented as mean values $\pm \mathrm{SD}$.

formulations. Thus, it was concluded that NVP leads to an increase in the rate of drug release and that delivery is seemingly unaffected by the microstructure due to the drug release being dominated by the PVP behavior. Nuances in this behavior are likely to depend on the affinity of the drug with NVP, PEGDA, and the oligomers and thus are complex and difficult to predict with a simple assessment of microstructure and/or affinity.

2.3. 3D Printing. 2.3.1. 3D Inkjet Printing Single DrugEluting Samples. Our screening showed that we could indeed influence the drug release rate either through our choice of solvent and its dissolution rate or through the composition of the material leading to phase separation or homogeneous structures. Consequently, for our 3D-printed samples, we subselected formulations that exemplified this behavior, as well as that offered by changing the core material and, thus, core polymer degradation rate. We then chose five materials, allowing us a direct comparison of these influences, and a route to demonstrating their efficacy in 3D printing: PCL-MA/ PEGDA, PTMC-MA/PEGDA, PCL-MA/PEGDA/PVP, PTMC-MA/PEGDA/PVP, and PLA-MA/PEGDA/PVP (Figure 3 ). We also incorporated a second active into these formulations to demonstrate the predictions' effectiveness for multiple actives. As the second active, we selected pitavastatin, which is a statin used for the treatment of high cholesterol. Pitavastatin offers an effective contrast since it has a similar $\log P$ to trandolapril (3.97 and 3.45, respectively) but different aqueous solubility ( 0.426 and $2.5 \mathrm{mg} / \mathrm{L}$, respectively). The amount of drug released from 3D-printed constructs was quantified on day $3,15,30$, and 60, and 3D OrbiSIMS was used to unambiguously identify each compound ${ }^{32}$ (Figure S6), and ToF-SIMS microscale resolved maps of bulk composition (via depth analysis) were used to understand the role of phase separation on drug distribution and release (Figure 3).

Examining the data, we can see that the use of PLA-MA/ PEGDA/PVP resulted in the fastest release. This result is most likely due to the combination of PLA being the fastest to degrade among all of the core materials ${ }^{33}$ and the ability of PVP to dissolve quickly in aqueous environments creating the conditions for rapid release. A very similar behavior was previously observed on polymer films of a PLA-g-PVP copolymer, where it was shown that degradation was accelerated by increasing the amount of PVP on the copolymer. This is due to the increased hydrophilicity by the addition of PVP and the channels generated after PVP dissolution which allowed more water entry through the sample. $^{34}$ For other formulations, adding PVP to the formulation was expected to result in increases in release. However, we see different results for PCL-MA and for PTMC$\mathrm{MA}$, which suggests that in $3 \mathrm{D}$-printed formulations the dominant lever was not necessarily the addition of PVP, but the microstructure obtained from the phase-separation behavior.

Comparing PCL-MA and PTMC-MA, we see that for PTMC-MA the addition of PVP resulted in a change in microstructure accompanied by a significant enhancement in drug release, but for PCL-MA, there was no change in microstructure, nor was there a significant difference in drug release profile. This is highlighted in our ToF-SIMS maps of the microstructure (Figure $3 \mathrm{c}$ ). We see that the addition of PVP to PTMC-MA results in a shift from a broadly homogeneous mixture of drug and polymer to a system where there is significant phase separation and network through which the drug can elute quickly. This phase 
separation and network is similarly observed for PCL-MA/ PEGDA, resulting in a similar release profile.

We thus conclude that all of these levers for controlling release are at play in our structures-where degradation and dissolution are fast-this can lead to rapid release, but that microstructure also plays a significant role in determining the rate of release. These observations provide us the tools by which a formulator can tailor implant composition by rational design and offers control over the release of multiple actives.

2.3.2. 3D Inkjet Printing of Multidrug Implants. Finally, we combined our two drug actives into two separated depots within the same cannular deliverable implant, the ACE inhibitor trandolapril (bottom) and the statin pitavastatin (top) (Figure 4), to demonstrate the targeting of combined treatment of coronary heart disease. ${ }^{35}$ In this case, we targeted relatively rapid release of our drugs over 15 days and so used PVP-containing polymer blends. We observed that release of both actives from our construct and that the drug release (Figure 4b) was in close agreement with that observed when released separately, demonstrating the effective manufacture of a tailorable multidrug implant, deliverable by conventional implantation procedures. We also confirmed with ToF SIMS the drugs' distribution through the entire bulk of the implant (Figure 4c).

\section{CONCLUSIONS}

We have demonstrated that we can control drug release through multiple mechanisms-choice of material, choice of reactive solvent, and by tailoring the composition to achieve predictable phase separation forming reproducible features on scales ranging from 1 to $50 \mu \mathrm{m}$. We have shown that the microstructure formed in our polymer blends was process dependent and arose as a function of the drop-by-drop deposition technique. As a result, we demonstrated that it is possible to functionally tailor the composition of 3D-printed constructs to successfully control the release of drugs incorporated within them. We selected suitable 3D-printing inks using complementary high throughput (HTP) methodologies that allowed us to screen for various desired properties and down select formulations from these screens. Screening of behavior in single drops using microscopy, AFM, and ToF SIMS, combined with the Flory-Huggins interaction parameter, provided a prediction of phase separation, and thus drug release, in 3D-printed structures. Consequently, we demonstrated a method that allows us to dial up desired drug release; we have created a library of material blends which can be selected to achieve their desired rate of release, and we have shown how they can be used to create a multidrug implant, meeting the demand for personalizable, degradable, and implantable polyimplants. In summary, this has resulted in a reliable toolkit for the predictable control and selection of behavior of 3D-printed constructs, and we have shown as an exemplar how this be used to control long-term drug release.

\section{EXPERIMENTAL SECTION}

4.1. Materials. D,L-lactide $99 \%$ was purchased from Alfa Aesar (by Thermo Fisher Scientific). Trimethylene carbonate (TMC) was purchased from polysciences. $\varepsilon$-caprolactone, extra dry dichloromethane (DCM), deuterated chloroform ( $\mathrm{CDCl} 3)$, acrylic acid, hydroxyethyl methacrylate (HEMA), benzyl alcohol (BA), poly (ethylene glycol) diacrylate 250, $\mathrm{N}$-vinyl-2-pyrrolidone (>99\%), 4dimethylaminopyridine, and triazabicyclodecene (TBD) were obtained from Sigma-Aldrich. Hexane, diethyl ether, $\mathrm{N}, \mathrm{N}$-dimethylfor- mamide (DMF), and methanol were obtained from Fisher. Trandolapril and pitavastatin were purchased from Carbosynth. In all cases, the vials were dried in an oven at $50{ }^{\circ} \mathrm{C}$ overnight prior to use, and HEMA and DCM were stored over molecular sieves and under an inert atmosphere.

4.2. Benzyl Alcohol (BA) and Hydroxyethyl Methacrylate (HEMA) Initiated Ring Opening Polymerization (ROP) of the Oligomers. The oligomers were synthesized by ring-opening polymerization using metal-free organocatalysis. ${ }^{21}$ ROP experiments were performed adopting "standard laboratory" conditions, i.e., ambient temperature and atmosphere. $-\mathrm{OH}$-ended macromers were initiated using BA; -MA and MA-A macromers were initiated using HEMA. Macromers were synthesized following the procedure by Ruiz-cantu et al. ${ }^{21}$ Briefly, $1000 \mathrm{mg}$ of cyclic monomer (caprolactone, lactide, or trimethylene carbonate) and BA or HEMA ([M]/[I] or $\mathrm{DP}_{0}$ ratios targeted to produce final molar masses of $5000 \mathrm{~g} / \mathrm{mol}$ were weighed into a vial, which had been dried in an oven at $50{ }^{\circ} \mathrm{C}$ overnight and capped with a rubber septum. DCM $(5 \mathrm{~mL})$ was then added via syringe, and the mixture was allowed to dissolve at room temperature (RT) for 5-10 min. Varying amounts of catalyst (1\% $\mathrm{mol} / \mathrm{mol}$ of TBD for lactide and trimethylene carbonate, $2 \% \mathrm{~mol} / \mathrm{mol}$ of TBD for caprolactone) were then added to trigger the ring-opening process. Reactions were observed to occur in time frames ranging from 15 to $120 \mathrm{~min}$, according to the monomer/initiator/solvent/ catalyst adopted ratios. The reaction was terminated by catalyst deactivation upon adding an acidic solution, and the polymer was purified by means of multiple precipitation steps and dried in a vacuum oven

4.3. MA-A Macromer End Capping. The MA-OH macromers were further functionalized with an acrylate end using a Steglich coupling esterification following the same procedure by Taresco, et $\mathrm{al}^{36}$ Briefly, PCLMA, PLAMA, or PTMCMA (0.2 mmol), and DMAP $(0.04 \mathrm{mmol})$ were added to DCM $(5 \mathrm{~mL})$ at room temperature in a glass vial under magnetic stirring until complete dissolution. A second solution was prepared by dissolving $1 \mathrm{mmol}$ of EDC and $1 \mathrm{mmol}$ of acrylic acid in $2 \mathrm{~mL}$ of DCM. After dissolution, both solutions were mixed. The reaction was allowed to stir for $48 \mathrm{~h}$. The modified macromers were purified under multiple precipitation steps and dried in a vacuum oven.

4.4. H NMR Spectroscopy. ${ }^{1} \mathrm{H}$ NMR spectra were recorded on a Bruker AV3400 $400.1 \mathrm{MHz}$ spectrometer using $\mathrm{CDC}_{13}$ as the solvent reference $(7.26 \mathrm{ppm})$. Chemical shifts are expressed in parts per million (d) downfield from internal standard tetramethyl silane.

PCLMA: ${ }^{1} \mathrm{H}$ NMR: $\left(400 \mathrm{MHz}, \mathrm{CDC}_{13}\right): \delta 6.14(\mathrm{~s}, 1 \mathrm{H}), 5.61(\mathrm{~s}$, $1 \mathrm{H}), 4.40-4.30(\mathrm{~m}, 4 \mathrm{H}), 4.08\left(\mathrm{t}, 2 \mathrm{H}^{*}[\mathrm{M}]:[\mathrm{I}]\right), 3.68(\mathrm{~m}, 2 \mathrm{H}), 2.33$ $\left(\mathrm{t}, 2 \mathrm{H}^{*}[\mathrm{M}]:[\mathrm{I}]\right), 1.97(\mathrm{~m}, 3 \mathrm{H}), 1.67\left(\mathrm{~m}, 4 \mathrm{H}^{*}[\mathrm{M}]:[\mathrm{I}]\right)$, and $1.41(\mathrm{~m}$, $\left.2 \mathrm{H}^{*}[\mathrm{M}]:[\mathrm{I}]\right)$.

PLAMA: ${ }^{1} \mathrm{H}$ NMR $\left(400 \mathrm{MHz}, \mathrm{CDC}_{\mathrm{l} 3}\right): \delta 6.14(\mathrm{~s}, 1 \mathrm{H}), 5.61(\mathrm{~s}$, $1 \mathrm{H}), 5.19\left(\mathrm{t}, 2 \mathrm{H}^{*}[\mathrm{M}]:[\mathrm{I}]\right), 4.36(\mathrm{~m}, 5 \mathrm{H}), 1.95(\mathrm{~m}, 3 \mathrm{H})$, and $1.59(\mathrm{~m}$, $\left.6 \mathrm{H}^{*}[\mathrm{M}]:[\mathrm{I}]\right)$.

PTMCMA: ${ }^{1} \mathrm{H}$ NMR $\left(400 \mathrm{MHz}, \mathrm{CDC}_{13}\right): \delta 6.14(\mathrm{~s}, 1 \mathrm{H}), 5.61(\mathrm{~m}$, $1 \mathrm{H}), 4.39(\mathrm{~m}, 4 \mathrm{H}), 4.25\left(\mathrm{~m}, 4 \mathrm{H}^{*}[\mathrm{M}]:[\mathrm{I}]\right), 3.74(\mathrm{~m}, 2 \mathrm{H}), 2.04(\mathrm{~m}$, $2 \mathrm{H} *[\mathrm{M}]:[\mathrm{I}])$, and $1.96(\mathrm{~m}, 3 \mathrm{H})$.

PCLMAA: ${ }^{1} \mathrm{H}$ NMR: $\left(400 \mathrm{MHz}, \mathrm{CDC}_{\mathrm{l} 3}\right): \delta 6.64(\mathrm{~s}, 1 \mathrm{H}), 6.29(\mathrm{~s}$, $1 \mathrm{H}), 6.14(\mathrm{~s}, 1 \mathrm{H}), 5.61(\mathrm{~s}, 1 \mathrm{H}), 5.59(\mathrm{~s}, 1 \mathrm{H}) 4.40-4.30(\mathrm{~m}, 4 \mathrm{H})$, $4.08\left(\mathrm{~m}, 2 \mathrm{H}^{*}[\mathrm{M}]:[\mathrm{I}]\right), 3.68(\mathrm{~m}, 2 \mathrm{H}), 2.33\left(\mathrm{~m}, 2 \mathrm{H}^{*}[\mathrm{M}]:[\mathrm{I}]\right), 1.97$ $(\mathrm{m}, 3 \mathrm{H}), 1.67\left(\mathrm{~m}, 4 \mathrm{H}^{*}[\mathrm{M}]:[\mathrm{I}]\right)$, and $1.41\left(\mathrm{~m}, 2 \mathrm{H}^{*}[\mathrm{M}]:[\mathrm{I}]\right)$.

PLAMAA: ${ }^{1} \mathrm{H}$ NMR $\left(400 \mathrm{MHz}, \mathrm{CDC}_{13}\right): \delta 6.64(\mathrm{~s}, 1 \mathrm{H}), 6.29(\mathrm{~s}$, $1 \mathrm{H}), 6.14(\mathrm{~s}, 1 \mathrm{H}), 5.61(\mathrm{~s}, 1 \mathrm{H}), 5.59(\mathrm{~s}, 1 \mathrm{H}), 5.19\left(\mathrm{~m}, 2 \mathrm{H}^{*}[\mathrm{M}]:[\mathrm{I}]\right)$, $4.36(\mathrm{~m}, 5 \mathrm{H}), 1.95(\mathrm{~m}, 3 \mathrm{H})$, and $1.59\left(\mathrm{~m}, 6 \mathrm{H}^{*}[\mathrm{M}]:[\mathrm{I}]\right)$.

PTMCMA: ${ }^{1} \mathrm{H}$ NMR $\left(400 \mathrm{MHz}, \mathrm{CDC}_{\mathrm{l}}\right): \delta 6.64(\mathrm{~s}, 1 \mathrm{H}), 6.29(\mathrm{~s}$, $1 \mathrm{H}), 6.14(\mathrm{~s}, 1 \mathrm{H}), 5.61(\mathrm{~s}, 1 \mathrm{H}), 5.59(\mathrm{~s}, 1 \mathrm{H}), 4.39(\mathrm{~m}, 4 \mathrm{H}), 4.25(\mathrm{~m}$, $\left.4 \mathrm{H}^{*}[\mathrm{M}]:[\mathrm{I}]\right), 3.74(\mathrm{~m}, 2 \mathrm{H}), 2.04 \mathrm{~m},(2 \mathrm{H} *[\mathrm{M}]:[\mathrm{I}])$, and $1.96(\mathrm{~m}$, $3 \mathrm{H})$.

4.5. Flory-Huggins Interaction Parameter. To investigate the phase separation in pin-printed droplets, we used a combination of the Flory-Huggins theoretical model and experimental characterization methods. The Flory-Huggins parameter $(\chi)$ describes the excess free energy of mixing and governs phase behavior for polymer blends and block copolymers. ${ }^{28}$ To calculate the $\chi$ value, we first 
obtained the Hansen solubility parameter of the individual components of the formulation using the HSPiP program, where $\delta d, \delta p, \delta h$, and $\delta_{\text {ТОт }}$ were obtained using the DYI tool of the software. We calculated the $\chi$ values following the procedure described by Imre et al..$^{37}$ using eq 1

$$
\chi=\frac{V_{\mathrm{r}}}{R T}\left(\delta_{1}-\delta_{2}\right)^{2}
$$

where $V_{\mathrm{r}}$ is the molar volume of the repeating unit of the oligomer, $R$ is the gas constant, $T$ is the absolute temperature, and $\delta_{1}$ and $\delta_{2}$ are the total solubility parameter $\left(\delta_{\mathrm{TOT}}\right)$ of the solvent and the oligomer, respectively.

4.6. Degradation. This study was performed on the 18 primary inks. Cast cylindrical samples with dimensions of $4 \mathrm{~mm}$ length and 1 $\mathrm{mm}$ radius were used for this test. The samples were prepared by transferring $15 \mu \mathrm{L}$ of each ink into a $4 \mathrm{~mm}$ piece of silicone tubing and then placed under UV for $3 \mathrm{~min}$ for crosslinking. Triplicates of each sample were prepared for each of the time points. Samples were transferred to individual vials containing $5 \mathrm{~mL}$ of phosphate-buffered saline solution and placed in an incubator at $37^{\circ} \mathrm{C}$. Triplicates of each sample were weighed, vacuum dried, and weighed again at week 1,4 , 8 , and 16 to calculate the mass loss at each time point.

4.7. Drug Release Quantification. The drug trandolapril was selected for this screening. Formulations containing $0.65 \% \mathrm{w} / \mathrm{v}$ of trandolapril were cast in the same way as for the degradation study. The dissolution method was adapted from the described method for the goserelin acetate implant on the FDA dissolution methods database. Briefly, samples were transferred to individual $20 \mathrm{~mL}$ scintillation vials containing $5 \mathrm{~mL}$ of phosphate-buffered saline solution $\mathrm{pH} 7.4$ and placed in an incubator at $37{ }^{\circ} \mathrm{C}$. Prior to sampling, the vials were removed from incubation and mechanically swirled with a digital orbital shake at $205 \mathrm{rpm}$ for $6 \mathrm{~s}$. At each time point, $500 \mu \mathrm{L}$ of the phosphate-buffered saline (PBS) solution was collected and filtered $(0.45 \mu \mathrm{m})$ for the high-performance liquid chromatography (HPLC) analysis. The PBS solution was refreshed at each time point. For the drug release studies of 3D-printed samples, the formulation was prepared in the exact same way as the cast ones.

4.8. HPLC. Samples were characterized with an Agilent (Santa Clara) HPLC Series 1260 system, equipped with an autosampler, degasser, UV lamp, and multidiode array detection. A wavelength of $210 \mathrm{~nm}$ was used to quantify trandolapril and $280 \mathrm{~nm}$ for pitavastatin. Method mobile phase compositions were $65 \%$ phosphate buffer and $35 \%$ acetonitrile (Fisher HPLC gradient grade). Phosphate buffer was composed of $6.8 \mathrm{~g} / \mathrm{L}$ monobasic potassium phosphate (anhydrous, Sigma-Aldrich) adjusted to $\mathrm{pH} 3.0$ with phosphoric acid (85-90\%, Fluka). An Ultimate LP-C18 column $(5 \mu \mathrm{m}, 25 \mathrm{~cm} \times 4.6 \mathrm{~mm}$ diameter) was used to separate the samples at $40{ }^{\circ} \mathrm{C}$. A flowrate of $1 \mathrm{~mL} / \mathrm{min}$ using a $10 \mu \mathrm{L}$ injection volume was implemented; runtime was $10 \mathrm{~min}$.

4.9. Cytotoxicity. To determine any evolving cytotoxicity of leached products, either through residual monomers or products emerging through polymer degradation, we performed a cytotoxicity test following the ISO 10993-5 standard. We used the extract test method; in this method, extracts are obtained by placing the test and control materials in separate cell culture media under standard conditions as follows. Triplicates of each formulation cast samples were sterilized under UV light $\left(0.05 \mathrm{~mW} / \mathrm{cm}^{2}, 265 \mathrm{~nm}\right)$ for $50 \mathrm{~min}$ and transferred into a 48-well plate. Each well containing a sample was filled with $1 \mathrm{~mL}$ of culture medium. Samples were incubated in the medium for a total of 30 days to allow for leaching of any cytotoxic components. After day 1, day 3, and day 30 of incubation, $200 \mu \mathrm{L}$ of the supernatant was transferred in triplicate to the cells seeded in 96well plates. BJ6 fibroblasts were grown in Dulbecco's modified Eagle's medium (DMEM) supplemented with $10 \%(\mathrm{v} / \mathrm{v})$ fetal calf serum, $1 \%$ minimum Eagle's essential medium (MEM) nonessential amino acids solution (Sigma-Aldrich), and $1 \%$ antibiotics/antimycotics (100 units $/ \mathrm{mL}$ penicillin, $100 \mathrm{mg} / \mathrm{mL}$ streptomycin, and $0.25 \mathrm{mg} / \mathrm{mL}$ amphotericin B; Life Technologies). Cells were cultured until they reached $80 \%$ confluency and subsequently detached from the culture surface using trypsin/ethylenediaminetetraacetic (EDTA) (0.25\%/
$0.02 \% \mathrm{w} / \mathrm{v}$ ), centrifuged at $200 \mathrm{~g}$ for $5 \mathrm{~min}$ and resuspended in culture medium. Cells were seeded in a 96-well plate at a density of 5000 cells per well and allowed to attach for $24 \mathrm{~h}$ before the cytotoxicity experiments. A new seeded well plate was used for each time point. Cells cultured in standard medium were used as a negative control. Cells were incubated for $24 \mathrm{~h}$ with the extracts. Cytotoxicity was measured using Presto Blue ${ }^{\mathrm{TM}}$ (Invitrogen) following the manufacturer's instructions. The fluorescent signal was measured with an automated microplate reader (Tecan) using an excitation wavelength of $560 \mathrm{~nm}$ and an emission wavelength of $590 \mathrm{~nm}$. For the cytotoxicity percentage calculations, the fluorescent background control was first subtracted from all of the samples. Then, the percentage was calculated by multiplying the fluorescence of each sample by 100 and then dividing the total by the average fluorescence of the negative control.

4.10. Printability Screening. To investigate the printability of the inks, we used a HT method developed by Zhou et al., ${ }^{38}$ where the viscosity and surface tension are measured using a liquid handler and the printability calculated using the Ohnesorge number $(Z=1 / \mathrm{Oh})$. The Ohnesorge number has been identified as the appropriate grouping of constants to characterize drop formation. ${ }^{39}$ Reis and Derby used a numerical simulation of drop formation to propose $10>$ $Z>1$ for stable drop formation. ${ }^{40}$ To identify printability at different temperatures, 18 inks formed by the combination of nine different macromers mixed with PEGDA and NVP were selected and screened in tempearture ranges from 40 to $70{ }^{\circ} \mathrm{C}$

4.11. Inkjet 3D Printing. The formulations were printed using a Dimatix Materials printer (DMP-2830 Fujifilm). The printer was enclosed in a metallic environment box and filled with nitrogen gas. A $10 \mathrm{pL}$ disposable printhead, Dimatix Materials Cartridge (DMC11610, Fujifilm), was used for printing. In-line UV curing was applied at the cartridge height immediately after each swath of ink droplets are deposited. The printing temperature was set to $40{ }^{\circ} \mathrm{C}$. The height of the printhead was set to $700 \mu \mathrm{m}$ with an increment of $9 \mu \mathrm{m}$ after each layer was printed. Single-drug sample dimensions were $5 \mathrm{~mm} \times$ $5 \mathrm{~mm} \times 1 \mathrm{~mm}$. Dual-implant sample dimensions were $2 \mathrm{~mm} \times 4 \mathrm{~cm}$ $\times 1 \mathrm{~mm}$. Individual sessile droplet size when deposited varied depending on the mixture being processed.

4.12. ToF SIMS. ToF SIMS of printed samples was carried out using a 3D OrbiSIMS (hybrid SIMS) instrument from IONTOF $\mathrm{GmbH}$ (Muenster, Germany). Secondary ion mass spectra were acquired in negative ion polarity with delayed extraction mode using a $30 \mathrm{keV} \mathrm{Bi3}{ }^{+}$primary ion beam delivering $0.3 \mathrm{pA}$. The ToF analyzer was set with $200 \mu$ s cycle time, resulting in a mass range between 0 and 3493 mass units. For the surface spectra, the primary ion beam was raster scanned over different areas with the total ion dose kept under the static limit of 1013 ions $/ \mathrm{cm}^{2}$. The 3D depth profiling data were acquired in dual-beam mode by raster scanning the primary ion beam over regions of up to $150 \mu \mathrm{m} \times 150 \mu \mathrm{m}$ at the center of $300 \mu \mathrm{m}$ $\times 300 \mu \mathrm{m}$ sputter craters formed using an argon gas cluster ion beam (GCIB). The GCIB was operated with $20 \mathrm{keV}$, and 2000 atoms in the cluster delivering a pulsed $5 \mathrm{nA}$ beam current. The analysis was performed in the "noninterlaced" mode with a low-energy $(20 \mathrm{eV})$ electron flood gun employed to neutralize charge build up. Three sputter frames were performed per cycle with 15 analysis scans per cycle and a pause time in between cycles of $0.5 \mathrm{~s}$. Optical profilometry was used to determine the crater depth after ToF-SIMS depth profiling experiments and calibrate the depth scale. Scans were obtained using a Zeta-20 optical microscope (Zeta Instruments, CA). All maps were produced using SurfaceLab, and 3D visualizations were produced using the simsMVA software. ${ }^{41}$ Intensities were normalized by total ion counts to correct for topographic features. The final 3D representations were created by combining rendered isosurfaces ranging from 40 to $90 \%$ of the maximum normalized intensity for each ion.

OrbiSIMS of a cross section of a multi-layer-printed sample containing all compounds of interest was carried out using a 3D orbiSIMS (hybrid SIMS) instrument. ${ }^{32}$ A $20 \mathrm{keV}$ Ar3000+ imaging GCIB of $5 \mu \mathrm{m}$ diameter was used as primary ion beam, delivering 18 pA (with duty cycle set to $37.7 \%$ ). Images were acquired over an area 
of $263 \mu \mathrm{m} \times 263 \mu \mathrm{m}$ using random raster mode. Optimal target potential was set to $-292 \mathrm{~V}$. Argon gas flooding was used to aid charge compensation. The images were collected in negative polarity, in the mass range of $75-1125 \mathrm{~m} / z$. The injection time was set to 511 ms. Mass resolving power was 243000 at $200 \mathrm{~m} / z$.

4.13. Statistical Analysis. Statistical analysis was performed using Prism version 8.0 (GraphPad Software). Statistical significance was determined by two-way ANOVA followed by a Tukey test. Plots are means with error bars indicating the standard error. Statistically significant values are presented as $* p<0.05$, **p $<0.01$, ***p $<$ 0.001 , and $* * * * p<0.0001$.

\section{ASSOCIATED CONTENT}

\section{SI Supporting Information}

The Supporting Information is available free of charge at https://pubs.acs.org/doi/10.1021/acsami.1c07850.

H NMR spectrum of macromers; microscopy images, topographical AFM images, and ToF SIMS images of the 18 formulations; Flory-Huggins interaction parameter calculations and values; images of cast samples in vitro degradation; viscosity and surface tension measurements; $z$-parameter values; biocompatibility test; and ToF SIMS and OrbiSIMS spectra peaks (PDF)

\section{AUTHOR INFORMATION}

\section{Corresponding Authors}

Derek J. Irvine - Centre for Additive Manufacturing, Faculty of Engineering, University of Nottingham, Nottingham NG7 2RD, U.K.; (1) orcid.org/0000-0003-1461-9851;

Email: Derek.Irvine@nottingham.ac.uk

Ricky D. Wildman - Centre for Additive Manufacturing, Faculty of Engineering, University of Nottingham, Nottingham NG7 2RD, U.K.; (1) orcid.org/0000-00032329-8471; Email: Ricky.Wildman@nottingham.ac.uk

\section{Authors}

Laura Ruiz-Cantu - Centre for Additive Manufacturing, Faculty of Engineering, University of Nottingham, Nottingham NG7 2RD, U.K.; (1) orcid.org/0000-00019763-8972

Gustavo F Trindade - School of Pharmacy, University of Nottingham, Nottingham NG7 2RD, U.K.

Vincenzo Taresco - School of Chemistry, University of Nottingham, Nottingham NG7 2RD, U.K.; 이이.org/ 0000-0003-4476-8233

Zuoxin Zhou - Centre for Additive Manufacturing, Faculty of Engineering, University of Nottingham, Nottingham NG7 2RD, U.K.

Yinfeng He - Centre for Additive Manufacturing, Faculty of Engineering, University of Nottingham, Nottingham NG7 2RD, U.K.

Laurence Burroughs - School of Pharmacy, University of Nottingham, Nottingham NG7 2RD, U.K.

Elizabeth A. Clark - Centre for Additive Manufacturing, Faculty of Engineering, University of Nottingham, Nottingham NG7 2RD, U.K.

Felicity R.A.J. Rose - School of Pharmacy, University of Nottingham, Nottingham NG7 2RD, U.K.; 이이.org/ 0000-0001-6640-8840

Christopher Tuck - Centre for Additive Manufacturing, Faculty of Engineering, University of Nottingham, Nottingham NG7 2RD, U.K.
Richard Hague - Centre for Additive Manufacturing, Faculty of Engineering, University of Nottingham, Nottingham NG7 $2 R D, U . K$.

Clive J. Roberts - School of Pharmacy, University of Nottingham, Nottingham NG7 2RD, U.K.; 1 orcid.org/ 0000-0001-9443-3445

Morgan Alexander - School of Pharmacy, University of Nottingham, Nottingham NG7 2RD, U.K.; (1) orcid.org/ 0000-0001-5182-493X

Complete contact information is available at:

https://pubs.acs.org/10.1021/acsami.1c07850

\section{Author Contributions}

The manuscript was written with the contributions of all authors. The majority of the experimental and characterization work was conducted by L.R.C. with 3D-printing support from Y.H., E.A.C., R.W., C.T., and R.H.; chemical synthesis support from V.T. and D.J.I.; and cell culture facilities and guidance provided by F.R.A.J.R. Material characterization was supervised by C.J.R. and D.J.I., printability screening performed by Z.Z., and the microarray printing performed L.R.C. with support from L.B. and overseen by R.D.W. and M.A. OrbiSIMS work was performed by G.T.F. supervised by C.J.R. The work was conceived and organized by D.J.I. and R.D.W.

\section{Funding}

This work was principally funded by the Engineering and Physical Sciences Research Council grant EP/N024818/1. Additional support came from EP/P031684/1 (GT) and EP/ N006615/1 (VT, LB), EP/P029868/1 (GT). Open access funding was provided by UK Research and Innovation.

\section{Notes}

The authors declare no competing financial interest.

\section{ACKNOWLEDGMENTS}

The authors thank Paul Gellert (AstraZeneca) for the useful discussions regarding drug delivery depots, their formulation, and needs for the future. The authors also thank Lyudmila Turyanska for the useful discussions about the data organization.

\section{REFERENCES}

(1) Sadée, W.; Dai, Z. Pharmacogenetics/Genomics and Personalized Medicine. Hum. Mol. Genet. 2005, 14, 207-214.

(2) Evans, W. E.; Relling, M. V. Moving towards Individualized Medicine with Pharmacogenomics. Nature 2004, 429, 464-468.

(3) Sun, W.; Lee, J.; Zhang, S.; Benyshek, C.; Dokmeci, M. R.; Khademhosseini, A. Engineering Precision Medicine. Adv. Sci. 2019, 6, No. 1801039.

(4) Khaled, S. A.; Burley, J. C.; Alexander, M. R.; Yang, J.; Roberts, C. J. 3D Printing of Five-in-One Dose Combination Polypill with Defined Immediate and Sustained Release Profiles. J. Controlled Release 2015, 217, 308-314.

(5) Boehm, R. D.; Miller, P. R.; Daniels, J.; Stafslien, S.; Narayan, R. J. Inkjet Printing for Pharmaceutical Applications. Mater. Today 2014, 17, 247-252.

(6) Khaled, S. A.; Alexander, M. R.; Wildman, R. D.; Wallace, M. J.; Sharpe, S.; Yoo, J.; Roberts, C. J. 3D Extrusion Printing of High Drug Loading Immediate Release Paracetamol Tablets. Int. J. Pharm. 2018, 538, 223-230.

(7) Elkasabgy, N. A.; Mahmoud, A. A.; Maged, A. 3D Printing: An Appealing Route for Customized Drug Delivery Systems. Int. J. Pharm. 2020, 588, No. 119732. 
(8) Boetker, J.; Water, J. J.; Aho, J.; Arnfast, L.; Bohr, A.; Rantanen, J. Modifying Release Characteristics from 3D Printed Drug-Eluting Products. Eur. J. Pharm. Sci. 2016, 90, 47-52.

(9) Wu, W.; Zheng, Q.; Guo, X.; Sun, J.; Liu, Y. A Programmed Release Multi-Drug Implant Fabricated by Three-Dimensional Printing Technology for Bone Tuberculosis Therapy. Biomed. Mater. 2009, 4, No. 065005.

(10) Allahham, N.; Fina, F.; Marcuta, C.; Kraschew, L.; Mohr, W.; Gaisford, S.; Basit, A. W.; Goyanes, A. Selective Laser Sintering 3D Printing of Orally Disintegrating Printlets Containing Ondansetron. Pharmaceutics 2020, 12, No. 110.

(11) Clark, E. A.; Alexander, M. R.; Irvine, D. J.; Roberts, C. J.; Wallace, M. J.; Sharpe, S.; Yoo, J.; Hague, R. J. M.; Tuck, C. J.; Wildman, R. D. 3D Printing of Tablets Using Inkjet with UV Photoinitiation. Int. J. Pharm. 2017, 529, 523-530.

(12) Darney, P.; Patel, A.; Rosen, K.; Shapiro, L. S.; Kaunitz, A. M. Safety and Efficacy of a Single-Rod Etonogestrel Implant (Implanon): Results from 11 International Clinical Trials. Fertil. Steril. 2009, 91, 1646-1653.

(13) Smith, L.; Mosley, J.; Johnson, J.; Nasri, M. Probuphine (Buprenorphine) Subdermal Implants for the Treatment Of OpioidDependent Patients. Pharm. Ther. 2017, 42, 505-508.

(14) Malclès, A.; Dot, C.; Voirin, N.; Vié, A.-L.; Agard, É.; Bellocq, D.; Denis, P.; Kodjikian, L. Safety of intravitreal dexamethasone implant (ozurdex): the safodex Study. Incidence and Risk Factors of Ocular Hypertension. Retina 2017, 37, 1352-1359.

(15) Citrin, D. L.; Resnick, M. I.; Guinan, P.; Al-Bussam, N.; Scott, M.; Gau, T. C.; Kennealey, G. T. A Comparison of Zoladex and DES in the Treatment of Advanced Prostate Cancer: Results of a Randomized, Multicenter Trial. Prostate 1991, 18, 139-146.

(16) Kamaly, N.; Yameen, B.; Wu, J.; Farokhzad, O. C. Degradable Controlled-Release Polymers and Polymeric Nanoparticles: Mechanisms of Controlling Drug Release. Chem. Rev. 2016, 116, 26022663.

(17) Fda. NEXPLANON (Etonogestrel Implant) Label 2015.

(18) De Nijs, H. Contraceptive Implant. EP0303306A11989.

(19) Pàmies, P.; Stoddart, A. Materials for Drug Delivery. Nat. Mater. 2013, 12, No. 957.

(20) Ulery, B. D.; Nair, L. S.; Laurencin, C. T. Biomedical Applications of Biodegradable Polymers. J. Polym. Sci. B. Polym. Phys. 2011, 49, 832-864.

(21) Ruiz-Cantu, L. A.; Pearce, A.; Burroughs, L.; Bennet, T.; Vasey, C.; Wildman, R.; Irvvine, D.; Alexander, C.; Taresco, V. Synthesis of Methacrylate-Terminated Block Copolymers with Reduced Transesterification by Controlled Ring-Opening Polymerization. Macromol. Chem. Phys. 2019, 220, No. 1800459.

(22) Fukushima, K. Biodegradable Functional Biomaterials Exploiting Substituted Trimethylene Carbonates and Organocatalytic Transesterification. Polym. J. 2016, 48, 1103-1114.

(23) Oh, H. J.; Freeman, B. D.; McGrath, J. E.; Ellison, C. J.; Mecham, S.; Lee, K.-S.; Paul, D. R. Rheological Studies of Disulfonated Poly(Arylene Ether Sulfone) Plasticized with Poly(Ethylene Glycol) for Membrane Formation. Polymer 2014, 55, 1574-1582.

(24) Arrieta, M. P.; López, J.; Rayón, E.; Jiménez, A. Disintegrability under Composting Conditions of Plasticized PLA-PHB Blends. Polym. Degrad. Stab. 2014, 108, 307-318.

(25) Sharma, P.; Kanchan, D. K. A Comparison of Effect of PEG and EC Plasticizers on Relaxation Dynamics of PEO-PMMA-AgNO3 Polymer Blends. Ionics 2013, 19, 1285-1290.

(26) Folttmann, H.; Quadir, A. Polyvinylpyrrolidone (PVP) - One of the Most Widely Used Excipients in Pharmaceuticals: An Overview. Drug Delivery Technol. 2008, 8, 22-27.

(27) Louzao, I.; Koch, B.; Taresco, V.; Ruiz Cantu, L.; Irvine, D. J.; Roberts, C. J.; Tuck, C. J.; Alexander, C.; Hague, R. J. M.; Wildman, R. D.; Alexander, M. R. Identification of Novel 'Inks' for 3D Printing Using High Throughput Screening: Bioresorbable Photocurable Polymers for Controlled Drug Delivery. ACS Appl. Mater. Interfaces 2018, 6841-6848.
(28) Xue, L.; Zhang, J.; Han, Y. Phase Separation Induced Ordered Patterns in Thin Polymer Blend Films. Prog. Polym. Sci. 2012, 37, 564-594.

(29) Kozuch, D. J.; Zhang, W.; Milner, S. T. Predicting the FloryHuggins $\chi$ Parameter for Polymers with Stiffness Mismatch from Molecular Dynamics Simulations. Polymers 2016, 8, No. 241.

(30) Díaz de los Ríos, M.; Hernández Ramos, E. Determination of the Hansen Solubility Parameters and the Hansen Sphere Radius with the Aid of the Solver Add-in of Microsoft Excel. SN Appl. Sci. 2020, 2, No. 676.

(31) Wang, F.; Altschuh, P.; Ratke, L.; Zhang, H.; Selzer, M.; Nestler, B. Progress Report on Phase Separation in Polymer Solutions. Adv. Mater. 2019, 31, No. 1806733.

(32) Passarelli, M. K.; Pirkl, A.; Moellers, R.; Grinfeld, D.; Kollmer, F.; Havelund, R.; Newman, C. F.; Marshall, P. S.; Arlinghaus, H.; Alexander, M. R.; West, A.; Horning, S.; Niehuis, E.; Makarov, A.; Dollery, C. T.; Gilmore, I. S. The 3D OrbiSIMS - Label-Free Metabolic Imaging with Subcellular Lateral Resolution and High Mass-Resolving Power. Nat. Methods 2017, 14, 1175-1183.

(33) Nair, L. S.; Laurencin, C. T. Biodegradable Polymers as Biomaterials. Prog. Polym. Sci. 2007, 32, 762-798.

(34) Peng, C.; Chen, H.; Wang, J.; Chen, Z.; Ni, M.; Chen, Y.; Zhang, J.; Yuan, T. Controlled Degradation of Polylactic Acid Grafting N-Vinyl Pyrrolidone Induced by Gamma Ray Radiation. J. Appl. Polym. Sci. 2013, 130, 704-709.

(35) Athyros, V. G.; Mikhailidis, D. P.; Papageorgiou, A. A.; Bouloukos, V. I.; Pehlivanidis, A. N.; Symeonidis, A. N.; Elisaf, M. Effect of Statins and ACE Inhibitors Alone and in Combination on Clinical Outcome in Patients with Coronary Heart Disease. J. Hum. Hypertens. 2004, 18, 781-788.

(36) Taresco, V.; Suksiriworapong, J.; Styliari, I. D.; Argent, R. H.; Swainson, S. M. E.; Booth, J.; Turpin, E.; Laughton, C. A.; Burley, J. C.; Alexander, C.; Garnett, M. C. New: N -Acyl Amino AcidFunctionalized Biodegradable Polyesters for Pharmaceutical and Biomedical Applications. RSC Adv. 2016, 6, 109401-109405.

(37) Imre, B.; Renner, K.; Pukánszky, B. Interactions, Structure and Properties in Poly(Lactic Acid)/Thermoplastic Polymer Blends. eXPRESS Polym. Lett. 2014, 8, 2-14.

(38) Zhou, Z.; Ruiz Cantu, L.; Chen, X.; Alexander, M. R.; Roberts, C. J.; Hague, R.; Tuck, C.; Irvine, D.; Wildman, R. High-Throughput Characterization of Fluid Properties to Predict Droplet Ejection for Three-Dimensional Inkjet Printing Formulations. Addit. Manuf. 2019, 29, No. 100792.

(39) Fromm, J. E. Numerical Calculation of the Fluid Dynamics of Drop-on-Demand Jets. IBM J. Res. Dev. 1984, 28, 322-333.

(40) Reis, N.; Derby, B. Ink Jet Deposition of Ceramic Suspensions: Modeling and Experiments of Droplet Formation. MRS Proc. 2000, 625, No. 117.

(41) Trindade, G. F.; Abel, M. L.; Watts, J. F. SimsMVA: A Tool for Multivariate Analysis of ToF-SIMS Datasets. Chemom. Intell. Lab. Syst. 2018, 182, 180-187. 